Journal Article

\title{
A Gaussian Process Surrogate Model Assisted Evolutionary Algorithm for Medium Scale Expensive Black Box Optimization Problems
}

Liu, B., Q. Zhang, Q. and Gielen, G.

This article is published by IEEE. The definitive version of this article is available at http://ieeexplore.ieee.org/xpls/abs all.jsp?arnumber $=6466380$

Recommended citation:

Liu, B., Q. Zhang, Q. and Gielen, G. (2013), 'A Gaussian Process Surrogate Model Assisted Evolutionary Algorithm for Medium Scale Expensive Black Box Optimization Problems', IEEE Transactions on Evolutionary Computation, Vol.18, No.2, pp.180-192. doi: 10.1109/TEVC.2013.2248012 


\title{
A Gaussian Process Surrogate Model Assisted Evolutionary Algorithm for Medium Scale Expensive Optimization Problems
}

\author{
Bo Liu, Qingfu Zhang, Senior Member, IEEE, and Georges Gielen, Fellow, IELE
}

\begin{abstract}
Surrogate model assisted evolutionary algorithms (SAEAs) have recently attracted much attention due to the growing neel for computationally expensive optimization in many reul-world applications. Most current SAEAs, however, focus on small-scale problems. SAEAs for medium-scale problems (i.e. 20-50 decision variables) have not yet been well studied. In this paper, a Gaussian process surrogate model assisted evolutionary algorithm for medium-scale computationally expensive optimization problems (CPEME) is proposed and investigated. Its major components are a surrogate model-aware search mechanism for expensive optimization problems when a high-guality surrogate model is difficult to build, and dimension reduction techniques for tackling the "curse of dimensionality". $A$ new framework is developed and used in GPEME, which carefully coordinates the surrogate modeling and the evolutionary search, so that the search can focus on a small promising area and is supported by the constructed surrogate model. Sammon mapping is introduced (1) transform the decision variables from tens of dimensions to a few dimensions, in order to take advantage of Gaussian process surrogate modeling in a low-dimensional space. Empirical studles on benchmark problems with 20, 30 and 50 variables and a real-world power amplifier design automation problem with 17 variables show the high efficiency and effectiveness of GPLME. Compared to three state-ol-the-art SAEAs. better or similar solutions can be obtained with $12 \%$ to $50 \%$ exact function evaluations.
\end{abstract}

Index Terms $\rightarrow$ surrogate model assisted evolutionary computation, surrogate models, expensive optimization, Gaussian process, prescreening, space mapping, dimension reduction.

\section{INTRODUCTION}

Many real-world optimization problems require expensive computer or physical simulation for evaluating their candidate solutions [1], [2]. Often, traditional gradient-based mathematical programming methods eannot be applied directly to these problems since analytic formulations are unavailable. Livolutionary algorithms (EA) cannot directly solve them either sinee a large number of function evaluations are unaffordable. Surrogate model assisted evolutionary algorithms (SALAs) are reeent promising approaches for dealing with such expensive optimization problems. SALAs use surrogate models to replace computationally expensive real function evaluattions. Since surrogate modeling and prediction/prescreening

The research of B. Lifu was supported by the Humboldt research lellowship, Germany and at scholarship of Katholieke Uniwersiteit Lecuven. Belgium.

B. Liu is with Department of Computing, Glyndwr University. Wrexhmo.

U.K. (e-mail: b.liu@glyndwr.ac.uk. liu_bo765 (3) yohoo.com.con).

Q. Zhan! is with the School of Computer Science and Electronic Engineer-

ing. University of Essex. CO4 3SQ. U.K. (e-minil: c/hamg (a)essex.ac.uk).

G. Gielen is with ESAT-MICAS, Katholicke Iniversiteit I.euven. Leuven.

Belgim (e-mail: Georges.Giclen (e) sat.kuleuven.be). use much less computational overhead than directly using the computationally expensive exact function evaluator, the computational cost can be reduced significantly.

This paper focuses on expensive optimization problems of 20) to 50 decision variables (medium-scale) and we assume that the computational budget available is in the range of 1,000 real function evaluations. Such problems can be found in many real-world applications such as mm-wave integrated circuit design [3], antenna design [4]. mechanical engineering [5] and manufacturing engineering [6]. For example, a single simulation of a high-frequency integrated circuit needs about 1) to 15 minutes, and a typical $T H \%$ computational electromagnetic simulation may cost 20-30 minutes. Due to the very tight time-to-market requirements, the optimization task should be eompleted within one to two weeks. Thus, one has to deliver the final solution within 1.000 function evaluations. Many of these computationally very expensive problems have around 20 to 50 design variables [3]. [4]. [0].

Most current SALAs focus on small-scale expensive oplimization problems [7], [8]. 19]. For problems with 20 or 30 variables. some successful attempts include [1]. [10] and [11]. [1] uses a weighted sum of the predicted values of different surrogate models to assist an LA, and the weights are adaptively adjusted based on the prediction uncertainty of different surrogate models. Reasonably good results on benchmark problems with 30 decision valriables have been obtained, but it cost 8,000 exact function evaluations. 111] uses the (jaussian process (GP) model with probability of improvement prescreening [12] as a global surrogate model and Lamarckian evolution (using the radial basis function) as a local surrogate model to accelerate an LA. Good results on benchmark problems with 20) variables ean be obtained with (6,000) exact function evaluations. [10] investigates (iP-based locial surrogate models and different prescreening methods to atsist $(\mu+\lambda)$ evolution strategies. Benchmark problems with 20 variables have been tested. and promising results have been obtained on some problems with only $1.0(0)$ exact function evaluations. However, its solution quality (especially for multimodal problems) needs to be improved. We believe that much effort should be made to develop efficient and effective SAEAs for medium-seale computationally expensive optimization problems to meet the increasing industry requirements.

Many surrogate models have been introduced to assist LAs. Among them. the (il modeling. response surface methods. artificial neural networks, support vector machines. and radial 
basis functions exhibit good performances and are widely used [7]. [8], [12], [10], [13], [14], [15], [16]. The GP modeling with the lower confidence bound (LCB) [17] prescreening is used in this paper, mainly based on the following considerations:

- (iP modeling is a theoretically sound method for determining a much smaller number of free model parameters than many other surrogate modeling approaches [18]. [19].

- (iP modeling can provide an estimate of the model uneertainty to each predicted point, which can be interpreted in a very natural way $[18],[19]$.

- Several prescreening methods are available for the (iP modeling in optimization. For eximple. the expected improvement presereening [7] has demonstrated its ability to escape a locally optimal area [1(0].

A key issue in an SALA is how to use a reasonable amount of computational effort to build a good model for locating the most promising candidate solutions. (iP modeling can involve heavy computational overheads. The computational complexity of a typical learning algorithm for GP modeling is $O\left(N_{i t} K^{3} d\right)$ [I0]. where $N_{i t}$ is the number of iterations. $d$ is the number of variables, and $K$ is the number of training data points. When $d$ is large, a large $N_{i t}$ is often required to obtain a good model. Our pilot experiments have shown that when using 150 training data, it can take 240-400) seconds to build a single (il) model for an objective function with 5() variables when the Blind DACE toolbox [20] is used on a Xeon 2.6() $\mathrm{GH} \%$ computer with the MATLAB environment. The cost will increase cubically as $K$ increases. An SAEA with a buclget of 1.000 function evaluations, as in this paper, may need to do (GP modeling for several hundred times. Although starting from the GP model from the previous iteration can reduce the computational overhead to some extent. it is still not realistic to use too many training points in the (jP modeling. On the other hand, using too few training data may deteriorate the reliability of the model and thus the solution quality. Besides the number of training data points, another important factor affecting the quality of a surrogate model is the location of the training data points. A standard LA. used as the search engine in most SALAs (c.g. [1]. [8]. [10]. 111]), generates new points mainly for optimization purpose but not for modeling. When an SALA with such an EA presereens a candidate solution, the model could be poor to judge the quality of this solution properly, sinee it is very likely that not enough training data points used for modeling are close to this solution, particularly in a high dimensional space.

This paper proposes two techniques to address the above issue. One is to employ a dimension reduction technique to map the training data to a lower-dimensional space on which the GP modeling will be conducted. In this way, the quality of the model can be largely improved due to the redueed space and the computational cost of the modeling can be reduced significantly. The other technique is to focus the search on a promising subregion, which is achieved by a new surrogate model-aware search mechanism. A new SAEA method is then proposed that uses these two technicues. called
Gaussian process surrogate model assisted evolutionary algorithm for medium-scale computationally expensive optimization problems (GPLME). Experimental results on benchmark problems show that (iPLME, with $12 \%$ to $50 \%$ exact function evaluations, outperforms or performs similarly to some other state-of-the-art SALAs [1], [10]. [11]. We also report the result of GPEME for an mm-wave integrated circuit design optimi\%ation problem.

The remainder of this paper is organized as follows. Section II introduces the surrogate modeling technique used in our proposed algorithm. A new SALA framework and GP'LME are then presented in Section III. Section IV presents the experimental results of (iPEMLi on some commonly used benchmark problems and a real-world engineering problem. Comparisons with some state-of-the-art methods are also provided in this section. Concluding remarks are presented in Section V.

\section{THE SURROGNTE MOIDELING IN GPLML}

\section{A. Gaussian Process Modeling}

To model an unknown function $y \quad f(x), x \in R^{d}$. the (iP modeling assumes that $f(x)$ at any point $x$ is a Gaussian random variable $N\left(/ 1, a^{2}\right)$, where $\mu$ and $\theta$ are two constants independent of $x$. For any $x, f(x)$ is a sample of $f+f(x)$. where $f(x) \sim N\left(0, n^{2}\right)$. For any $x, x^{\prime} \in R^{\prime d}, c\left(x, x^{\prime}\right)$, the correlation between $c(x)$ and $c\left(x^{\prime}\right)$, depends on $x$. $x^{\prime}$. More preciscly,

$$
c\left(x, x^{\prime}\right) \quad \exp \left(\sum_{i=1}^{d} \theta_{i}\left|x_{i} \quad x_{i}^{\prime}\right|^{p_{i}}\right) .
$$

where parameter $1 \leq p_{i} \leq 2$ is related to the smoothness of $f(x)$ with respect to $x_{i}$, and parameter $\theta_{i}>0$ indicales the importance of $x_{i}$ on $f(x)$. More details about (jP modeling can be found in $|2|]$.

1) Ilyper Parameter Lstimation: (iiven $K$ points $r^{1}, \ldots x^{K} \in R^{d}$ and their $f$-function values $y^{1} \ldots \ldots y^{K}$, then the hyper parameters $\mu, \sigma, \theta_{1} \ldots, \theta_{d}$. and $p_{1} \ldots \ldots p_{d}$ can be estimated by maximizing the likelihood that $f(x) \quad y^{i}$ at $\left.x \quad x^{i}(i \quad 1, \ldots, K) \mid 7\right]$ :

$$
\frac{1}{\left(2 \pi 0^{2}\right)^{K / 2} \cdot \sqrt{\operatorname{det}(C)}} \exp \Gamma^{\Gamma} \frac{(y \quad \mu 1)^{T} C^{-1}(y \quad \mu 1)^{\top}}{20^{2}}
$$

where $C$ is a $K \times K$ matrix whose $(i, j)$-clement is $c\left(x^{i}, x^{j}\right)$. y) $\left(y^{1}, \ldots y^{K}\right)^{T}$ and 1 is a $K$-dimensional column vector of ones.

To maximize (2), the values of $\mu$ and $\alpha^{2}$ must be:

$$
\text { il } \frac{1^{T} C^{-1} y}{1^{T} C^{-1} 1}
$$

and

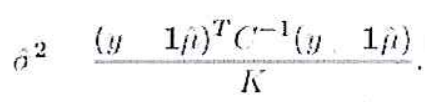

Substituting (3) and (4) into (2) eliminates the unknown parameters $\mu$ and $a$ from (2). As a result, the likelihood function depends only on $\theta_{i}$ and $p_{i}$ for $i \quad 1, \ldots, d,(2)$ can then be maximized to obtain estimates of $\theta_{i}$ and $j_{i}$. The estimates $\mu$ and $\delta^{2}$ ean then readily be obtained from (3) and (4). In our experiments, we use the MAILAB optimization toolbox to optimize the likelihood function. 
2) The Best Linear Unbiased Prediction and Predictive Distribution: Given the hyper parameter estimates $\dot{\theta}_{i}, \hat{p}_{i}, \hat{\mu}$ and $\hat{o}^{2}$. one can predict $y \quad f(x)$ at any untested point $x$ based on the $f$-function values $y^{i}$ at $x^{i}$ for $i \quad 1, \ldots K$. The best linear unbiased predictor of $f(x)$ is [7], [22]:

$$
f(x) \quad \beta+r^{T} C^{-1}(y \quad 1 \mu)
$$

and its mean squared error is:

$$
s^{2}(x) \quad \hat{o}^{2} \mid 1 \quad r^{T} C^{-1} r+\frac{\left(11^{T} C^{-1} r\right)^{2}}{1^{T} C^{-1} r} .
$$

where $r \quad\left(c\left(x, x^{1}\right), \ldots, c\left(x, x^{K}\right)\right)^{T} . N\left(f(x), s^{2}(x)\right)$ can be regarded as a predictive distribution for $f(x)$ given the function values $y^{i}$ at $x^{i}$ for $i \quad 1 \ldots, K$.

3) Lower Confidence Bound: We consider minimization of $f(x)$ in this paper. Given the predictive distribution $N\left(f(x), s^{2}(x)\right)$ for $f(x)$, the LCB of $f(x)$ can be defined as [17]:

$$
f_{l c h}(x) \quad f(x) \quad \omega s(x)
$$

where $\omega$ is a constant. In our algorithm, we use $f_{l e b}(x)$ instead of $f(x)$ itself to measure the quality of $x$. The use of LCB ean balance the search between promising areas (i.e., with low $f(x)$ values) and less explored areas (i.e., with high $s(x)$ values).

\section{B. Dimension Reduction}

A key issue in the design of an SALA can be stated as follows:

- Given $K$ points $x^{1} \ldots, x^{K} \in R^{d}$ and their $f$-function values $y^{1}, \ldots, y^{K}$. Let $x^{K+1} \ldots \ldots x^{K+M}$ be $M$ untested points in $R^{d}$. How ean one rank the $M$ untested points without exact function evaluation?

A natural solution to this issue is that one first builds a (il? model based on the exact evaluated $y^{i}$ data, computes the LCB values of each untested point and then ranks them based on their L.CB values. This procedure is so called prescreening. In GPLMLE, we only select the point with the best LCB value for exact function evaluation based on the obtained ranking. It is desirable that the selected point is a high-ranked one among all the untested points. Our pilot experiments have shown that for the test problems with 50 variables used in this paper, it is often very hard to achieve the above goal with 100 or 150 training data. Using a larger $K$. as argued in Section I, tan lead to a large amount of eomputational overhead in GP modeling.

To deal with problems with around 50 decision variables, we propose an efficient way to do presereening with dimension reduction. The key idea is to map the training data to a lowerdimensional space and then do GP modeling. As a result. the disadvantages caused by the insufficient number of training data points for medium-scale problems can be overeome. The method works as follows:

Prescreening by (GP with dimension reduction (GP+DR)

Input: (1) Training data: $x^{1}, \ldots, x^{K}$ and $y^{1}, \ldots, y^{K}$. (2) Points to prescreen: $f^{K+1} \ldots \ldots, x^{K+M}$.

Output: The estimated best solution among all the untested points $x^{K+1} \ldots \ldots x^{K+M}$.
Step 1: Map $x^{1} \ldots \ldots x^{K+M} \in R^{d}$ into $R^{l}$. where $l<$ d. and obtain their corresponding images in $R^{l}$ : $\bar{r}^{1} \ldots \ldots i^{K+M}$

Step 2: Build a (iP model by using the $i^{i}$ and $y^{i}$ data (i $1, \ldots, K$ ). and then use the model to compute the LCB value of each $x^{K+j}(j \quad 1, \ldots, M)$.

Step 3 : Find the point with the smallest LCB value among $i^{K+1}, \ldots, x^{K+M}$. Output its corresponding point in the original decision space $F^{d t}$ as the estimated best solution.

There are many machine learning methods which can transform the original space $R^{d}$ to the latent space $R^{l}$ for dimension reduction [23]. We have the following two considerations:

- The neighborhood relation among the data points plays a critical role in the correlation function in (il) modeling. Therefore, the neighborhood relationship anong the $t^{i}$ points in $R^{l}$ should be as similar as possible to those among all the $r^{i}$ points $(i \quad 1, \ldots, K-M)$ in $l^{i d}$. The pairwise distances among the data points should be preserved as much as possible.

- The mapping technique should not be very costly since it will be used many times in an SALA.

Based on these considerations, we use the Sammon mapping [24] which minimizes the differences between eorresponding inter-point distances in the two spaces. Moreover, it is not expensive when $K+M$ and $l$ are not large. Sammon mapping minimizes the following error function:

$$
\begin{aligned}
& E \overbrace{1 \leq i<j \leq K+M} \frac{1}{\operatorname{dis}\left(x^{i}, \mu^{i}\right)} \\
& \times \Gamma_{1 \leq i<j \leq K+M} \frac{\left[\operatorname{dis}\left(x^{i}, x^{j}\right) \operatorname{dis}\left(x^{i}, x^{j}\right)^{2}\right.}{\operatorname{dis}\left(x^{i}, x^{j}\right)}
\end{aligned}
$$

where $\operatorname{dis}(*, *)$ is the Luclidean distance.

Minimization of the error function in (8) is a large-scale quadratic optimization problem. In our experiments. we use the gradient descent method in [25] to solve it and we set 14 (more details ean be found in Section IV). We use the principle component analysis (PCA) technique to generate an initial point for the optimization process. When $K \quad 100$.

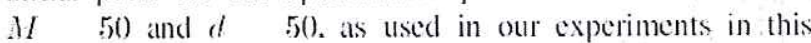
paper. the CPU time consumed by Sammon mapping is a few seconds on a $2.66(\mathrm{GH} \%$ computer.

\section{CiP Modeling with Dimension Reduction using Sammon Mapping is Direct (iP Modeling}

In the following, we discuss the major advantages and disadvantages of these two approaches.

GP modeling with dimension reduction (GP + DR):

- Advantages:

- Sammon mapping transforms the training data points from the original space $R_{i}^{d}$ to a lower-dimensional space $R^{l}$. Therefore, the number of training data points required for a good GP model can be reduced.

- Since the modeling is conducted in a lowerdimensional space. the computational overhead can also be reduced significantly. 
- Disadvantage:

- Although Sammon mapping minimizes the error function $E$ between the two spaces, the error cannot be zero. This means that some neighborhood information of the training data points in the original space will be lost in the latent space.

Direct GP modeling in the original space $R^{d}$ :

- Advantage:

- Errors caused by dimension reduction can be avoided.

- Disadvantages:

- In the case of large $d$ (such as $d \quad 5(0)$, it is often very difficult to select a sufficient number of proper training data points from the available data set to build a good GP model for a particular search area of interest.

- When $d$ is large. direct modeling will be costly as pointed out in Section 1.

For these reasons, we decide to use the direct (iP modeling in our proposed algorithm when $d \quad 20$ and 30 , and (GP+DR in the ease of $d$ 50. One thing that needs to be mentioned is that for problems with 20 or 30 variables, both methods can obtain reasonably good results (better than the available methods). This is related to our new SALA framework. which is another main contributor to GPEML and which will be described in the next section.

\section{THE PROPOSED GPEME AlgorithM}

\section{A. Differential Evolution (DE)}

The DE algorithm is used as the seareh engine in our proposed GPEML algorithm. DE: is an effective and popular global optimization algorithm. It uses a differential operator to create new candidate solutions [26]. There are quite a few different DE variants. In this paper. we use DE/best/I to generate new solutions for prescreening. The DE:/hest/1 mutation uses the current best solution as the base vector, so as to increase the speed of generating promising candidates.

Suppose that $P$ is a population and the best individual in $P$ is $x^{\text {best }}$. Let $x \quad\left(x_{1}, \ldots, x_{d}\right) \in R^{d}$ be an indiviclual solution in $P$. To generate a child solution $u \quad\left(u_{1} \ldots \ldots u_{d}\right)$ for $x$, DI: $/$ best/I works as follows.

A donor vector is first produced by mutation:

$$
v \quad x^{b c s t}+F \cdot\left(x^{r_{1}} \quad x^{r_{2}}\right)
$$

where $x^{r_{1}}$ and $x^{r_{2}}$ are two different solutions randomly selected from $P$ and are also different from $x^{\text {best }} . F \in(0,2]$ is a control parameter, often called the sealing factor [26]. Then the following crossover operator is applied to produce $u$ :

1 Randomly select a variable index $j_{\text {rand }} \in\{1, \ldots, d\}$,

2 For each $j \quad 1$ to $d$. generate a uniformly distributed random number rand from (0,1) and set:

$$
u_{j} \quad\left(\begin{array}{ll}
v_{j}, & \text { if }(\text { rand } \leq C R) \mid j \quad \text { irand } \\
x_{j}, & \text { otherwise }
\end{array}\right.
$$

where $C R \in[0,1]$ is a constant called the crossover rate.

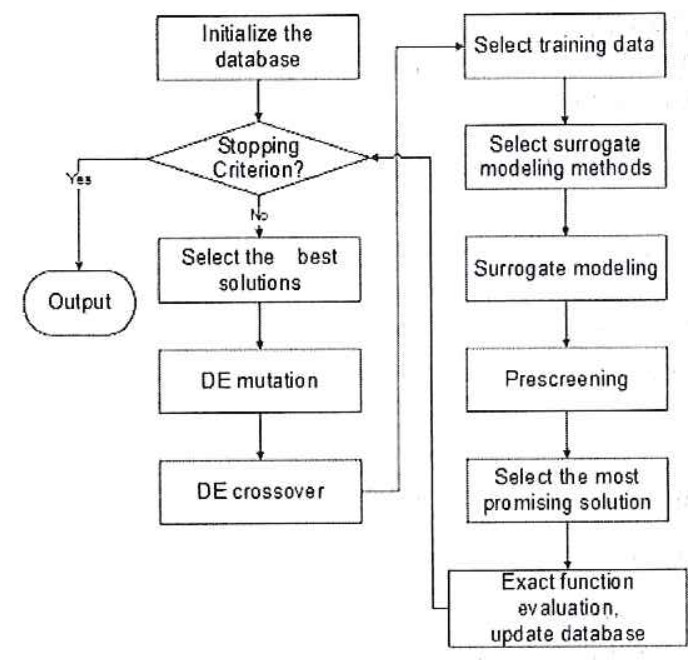

Fig. 1. The llow diagram of GPleME

\section{B. The GPLME Frumework}

Like most other SAEAs, (jl'LME reeords all the evaluated solutions and their function values in at database. Once an exact function evaluation has been conducted for a new solution $x$. this solution and its real function value $y$ will be added to the database. To initialize the database. a Design of Experiments method, Latin Hypercube sampling (L.HS) |27], is used to sample a set of initial points from the search space. The L.HS simpling method samples the design space more uniformly, and henee, ean use fewer samples to achieve a more effective sampling. It has been widely used for initialization in some other SAEAs [7], [15]. Let the search space be $\left[a, b^{d d}\right.$. The GlPLML algorithm works as follows (see lig. 1):

Step 1: Use LHS to sample a solutions from $f a, b^{d}$. evaluate the real function values of all these solutions and let them form the initial database.

Step 2: If a preset stopping criterion is met. output the best solution in the database: otherwise go to step 3 .

Step 3: Select the $\lambda$ best solutions (i.e.. with the lowest function values) from the database to form a population $P$.

Step 4: Apply the DE: operators on $P^{\prime}$ to generate $\lambda$ ehild solutions.

Step 5: Take the ; newest solutions in the database and their function values as the training data to presereen the $\lambda$ ehild solutions generated in Step + by using (il) in the original space or $\left(\mathrm{SP}^{2}+\mathrm{D}\right) \mathrm{R}$ with the $\mathrm{LCCB}$ prescreening.

Step 6: Evaluate the real function value of the estimated best child solution from Step 5. Add this evaluated solution and its function value to the database. (io back to Step 2.

The flow diagram of the Gl'LML framework is given in lig. 1

We ean make the following remarks on the (iPLiML framework: 
- The population P' generated in Step 3 consists of the $\lambda$ best solutions in the database. Most of these solutions may not be far away from each other, particularly after several iterations. Therefore, most child solutions generated in Step 4 are in a relatively small promising subregion.

- In each iteration. at most one new solution enters $P$ in Step 3. Hence. in several consecutive iterations. the solutions to be prescreened in Step 5, which are generated from $P$ in step 4 , should not be far away from each other: particularly, when most solutions in $P$ are distributed in a small region. For this reason, the training data points (obtained from Step (6) could not be far from a solution to be persereened, which is desirable for (ilPEML:

- The $r$ solutions in Step 5 are the most recently generated best candidate solutions. and it is reasonable to assume that these solutions are also not far alway from the current promising subregion. Thus the model built by using these solutions should be reasonably accurate for ranking the $\lambda$ child solutions generated in Step 4.

- It is not necessary to build a very accurate (jp model in Step 5 for prescreening. We only require that the GP model can help to select a reasonably good solution.

Taking the Ackley function with 20) variables as an example. in GPJ:ME (the experimental setting is given in the next section), the probabilities on 20 runs that the best ranked solution by prescreening in Step 5 is among the top $20 \%$ and $10 \%$ of the $\lambda$ child solutions in terms of exact function values are $86.1 \%$ and $75.8 \%$, respectively. Clearly, the GP modeling works very well in CiPLML.

To get a rough idea of the search ability of the GiPEML framework. we remove the GP modeling and prescreening. Instead. we concluct exact function evaluations to all the $\lambda$ child solutions in each iteration, and randomly select one from the top $\beta$ solutions. In such a way. we simulate the (iP) modeling and prescreening.

We set $a \quad 100$ and $\lambda \quad 50$. We have tested tive different 3 values: $1,3,5,7$ and 9 . The function values of the best solution found so far versus the number of iterations is plotted in lig. 2 . These results are based on 20 runs. It is clear from Fig. 2 that the convergenee speed decreases as the $\beta$ value increases. This indicates that the quality of the Gl' model does have an impact on the convergence speed of GPLME: When $1 \leq \beta \leq 7$. the best objective function value obtained with 1.000 iterations is very close to (), the global optimal value. This implies that the proposed GPEME framework works well if the best solution based on presereening is among the top-ranked candidates in the newly generated $\lambda$ candidate solutions in terms of exact function values.

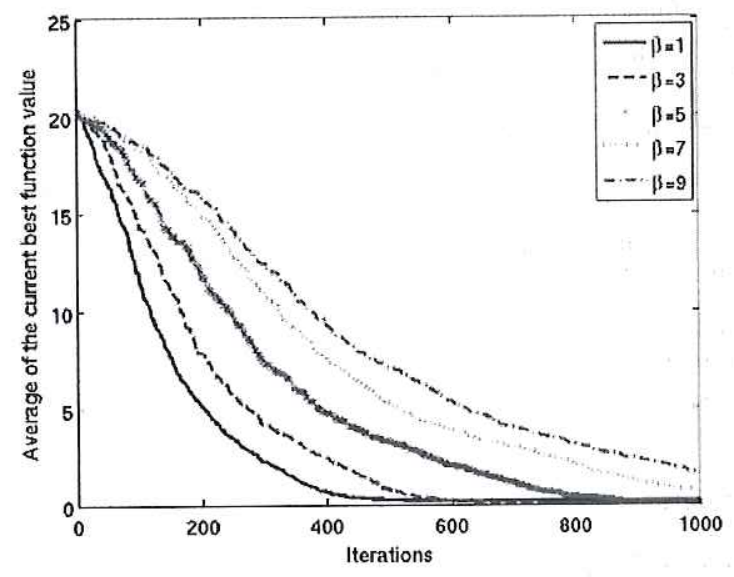

Fig. 2. Comvergence Curve in simulated (illente

\section{EXPERIMENTAL STUDIE:S}

\section{A. Parameter Settings}

There are several control parameters in (iPLEML. To set some parameters, we have conducted pilot experiments on benchmark problems. Their settings and our considerations are given as follows:

- The scaling factor $F$ and the crossover rate $C h$ in the DLi operators: The setting of the DL parameters has been well investigated. Following [26], we set $F$ to $(0.8$, and CR to 0.8 .

- The number of training data points $r$ in the GP modeling: The tradeoff between the model quality and the computational cost is eonsidered when setting \%. Our pilot experiments have shown that for medium-scale problems, when using less than 80 training data to construct the surrogate model, the selected best candidate based on the (il) model and prescreening is not a truly high-ranked candidate in many occasions. On the other hand. although the model quality can improve when using more training data, the computational cost of modeling also increases significantly. Here are some training times to construct a (jP model (using the Blind DACL toolbox |2(0) for the problems tested in this paper (see Appendix) on a Xeon 2.66GHz computer in the MATLAB environment. If 100 training data are used, for the problems with 20. 30 and 50 variables, the CPU time to construct a (iP model is about 10-20 seconds. 15-35 seconds, 120 to 250 seconds, respectively. When 150 training data ate used, the (CPU time increases to $3(0-50$ seconds. $8(0)-110$ seconds, and 240-400 seconds, respectively. We have found that $80 \leq r \leq 120$ can produce a reasonably good surrogate model for (iPLME with an aceptable amount of computational time. In our experiments using benchmark problems, $; \quad 100$ is used.

- The dimensionality $/$ of the low-dimensional space in Sammon mapping: Our pilot experiments have indicated that $l>6$ can make the search quite slow and $/ 2$ can lead to poor results. In our experiments. we sed $l$.

- $\omega$ used in LCB: Following [10]. |17]. $\omega 2$ 2 is used. 
- The number of initial samples $a$ in Step 1: [10] suggests that at least $2 d$ samples should be used to construct a reasonably good local GP model. However, the initial samples are randomly generated spreading all over the search space, so more samples are necessary. We use 100 initial samples in the experiments using benchmark problems.

- $\lambda$ in Step 3: Our pilot experiments on $\lambda \quad 20,30, \ldots, 80$ have shown that $30 \leq \lambda \leq 6()$ works well. A large $\lambda$ value causes slow convergence and a small value can easily lead to premature eonvergence. We set $\lambda \quad 50$ in the experiments using benchmark problems.

\section{B. Test Problems}

To compare with three state-of-the-art methods reported in [1]. [10]. [11], we take the problems used in their works ats the test instances. The test problems in [10], [11] have 20 variables. [10) uses the Sphere problem (simple unimodal). the Ellipsoid problem (unimodal), the Step problem (discontinuous) and the Ackley problem (multimodal). [11] uses the Sphere problem, the Rosenbrock problem (unimodal but with a narrow valley near the global optimum). the Ackley problem. the Griewank problem (multimodal) and the Rastrigin problem (multimodial). [1] uses test problems with 30 variables. including the Ackley problem. the Griewank problem. the Rosenbrock problem, two shifted rotated problems and five hybrid composition functions from [28]. Besides problems with 20 or 30 variables, we also test some problems with 50 variables, which have not been tested by the other methods. The test problems used in our experiments are listed in Table 1 and more details can be found in the Appendix.

We also test our method on a practical design optimization problem of an mm-wave power amplifier with 17 design variables.

\section{The CFPEME Performance and Comparisons with State-of- the-art SAEAs}

The statisties of the best function values obtained by GPEML with 1,00(0) function evaluations on 20 independent runs for [:]-1:14 are reported in Table II.

From Table II, for most problems with 20 and 30 variables. GPLME ean find very good solutions with 1,000 exact function evaluations. The exceptions are $\mathrm{F}_{4} 4$ and $\mathrm{F} 5$ (Rosenbrock problem with 20 and 30 variables). 213 and 1\%14. Although the Rosenbrock problem is unimodal, the narrow valley of the global optimum makes it difficult to optimize. F13 and Fil4 are artificially designed complex problems. The standard DE with $30,0(0)$ exact function evaluations cannot produce good results for $F 5,1: 13$ and 714 as shown in the next subsection. For the problems with 50 variables, although the obtained solutions are not very close to the global optimal solutions, their qualities are reasonably good.

We have compared GPEML with GS-SOMA II]. SAGAGLS 111] and MAES [10]. As shown in Table 1. GS-SOMA has been tested on F5. F8, F11, 1213 and 114 in 11]. SAGA(iLS on F4, 17 and 1:10 in [1]1, and MALS on F1 and 1:7 in [10]. Table IIl to Table $V$ report the following values:
TABLE I

TEST P'ROBIEMS USED IN THE EXPERIMENTAL. STUDIES

\begin{tabular}{|c|c|c|c|c|c|}
\hline Problem & $\begin{array}{l}\text { Objective } \\
\text { lunctions }\end{array}$ & $\begin{array}{l}\text { No. of } \\
\text { Viri- } \\
\text { ables }\end{array}$ & $\begin{array}{l}\text { Globial } \\
\text { opti- } \\
\text { mumi }\end{array}$ & P'roperty & $\begin{array}{l}\text { Comparisum } \\
\text { Method }\end{array}$ \\
\hline $\mathrm{Fl}$ & Fllipsoid & 20 & 0 & unimudal & [ाणा \\
\hline $\mathrm{F}_{2}$ & Ellipsoid & 30 & 0 & unimodial & N.A. \\
\hline $\mathrm{F} 3$ & Ellipsoid & 50 & 0 & unimotal & N.A. \\
\hline $\mathrm{F} 4$ & Rusenbrock & 20 & 0 & $\begin{array}{l}\text { unimodal } \\
\text { with } \\
\text { namrow } \\
\text { valley }\end{array}$ & 1111 \\
\hline F5 & Roscubrock & 30 & 0 & $\begin{array}{l}\text { unimodil } \\
\text { with } \\
\text { narrow } \\
\text { valley }\end{array}$ & $\mid 11$ \\
\hline $\mathrm{F} 6$ & Rosenbrock & 50 & 0 & $\begin{array}{l}\text { uninodial } \\
\text { with } \\
\text { mamrow } \\
\text { valley }\end{array}$ & N.A. \\
\hline $\mathrm{Fi}$ & Ackiley & 20 & 0 & multimodal & $\begin{array}{l}|10| . \\
|11|\end{array}$ \\
\hline F8 & Ackley & 30 & 0 & mulcimedial & [1] \\
\hline Fi) & Ackley & 50 & 0 & multinodial & N.A. \\
\hline F10 & Griewank & 20 & 0 & multinodal & 1111 \\
\hline$F 11$ & Griewank & 30 & 0 & multimodial & 111 \\
\hline $\mathrm{F} 12$ & Griewank & 50 & 0 & multimedial & N.A. \\
\hline $\mathrm{F} 13$ & $\begin{array}{l}\text { Shilted } \\
\text { Rotated } \\
\text { Rastrigin }\end{array}$ & 30 & -330 & $\begin{array}{l}\text { very } \\
\text { compli- } \\
\text { catcd } \\
\text { multi- } \\
\text { modal }\end{array}$ & $|1|$ \\
\hline $\mathrm{F} 14$ & $\begin{array}{l}\text { Rotated } \\
\text { Hybrid } \\
\text { Com- } \\
\text { position } \\
\text { Funstion } \\
\text { (F19 in } \\
\text { (11) }\end{array}$ & 30 & 10 & $\begin{array}{l}\text { very } \\
\text { compli- } \\
\text { ciated } \\
\text { multi- } \\
\text { modil }\end{array}$ & 111 \\
\hline
\end{tabular}

N.A. means that nene of the three methods $|1|$. $|110|$. $|11|$ has been tested on these instances.

TABI.F II

STATISTICS OF TIE BEST FUNCTION VALUES OBTAINED BY GPFML IN 1.000 EXACT FUNCTION EVALUATIONS FOR F1-F14

\begin{tabular}{|c|c|c|c|c|}
\hline Problem & best & worst & averatge & sitd \\
\hline $\mathrm{Fl}$ & $1.27 \mathrm{e}-6$ & $7.2 \mathrm{c}-5$ & $1.3 c-5$ & $2.18 \mathrm{e}-5$ \\
\hline 12 & 0.0155 & 0.1647 & 0.0762 & 0.0401 \\
\hline $\mathrm{F} 3$ & 134.0681 & 372.5567 & 221.0774 & 81,6123 \\
\hline F4 & 15.1491 & 75.8806 & 22.4287 & 18.7946 \\
\hline F5 & 26.2624 & 88.2325 & 46.1773 & 25.5140 \\
\hline Fo & 172.3547 & $401.418 ?$ & 258.2787 & $80.18 \%$ \\
\hline $\mathrm{F} 7$ & 0.0037 & 1.840 .3 & 0.1990 & $0.57 / 1$ \\
\hline F8 & 1.9491 & $4.96+10$ & 3.010105 & 0.9250 \\
\hline F) & 9.2524 & 14.9343 & 13.2327 & 1.5816 \\
\hline F10 & 0.0002 & 0.2234 & 0.03007 & $0.06 \mathrm{~s} 2$ \\
\hline FII & 0.7368 & 1.0761 & 0.9960 & 0.1080 \\
\hline $\mathrm{F} 12$ & 22.5456 & $6+.9767$ & $36.6+50$ & 13.1755 \\
\hline $\mathrm{F} 13$ & -57.06178 & 18.0327 & -21.8610 & 36.4192 \\
\hline F14 & 033.1601 & 9) 2.8618 & 958.59 .30 & $25.60)+6$ \\
\hline
\end{tabular}

(c) 2013 Crown Copyright. Personal use is permitted. For any other purposes, permission must be obtained from the IEEE by em ailing pubs-permissions aieee.org. 
TABLE III

COMPARISON OF GPEME WITH GS-SOMA [1]

\begin{tabular}{|c|c|c|c|}
\hline Priblem & (JPEME 18 est ${ }_{2918)}$ & 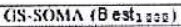 & (IS-SOMA ( H $_{2822)}$ \\
\hline F5 & th.1773 & 2981 & $\times *(x) 1$ \\
\hline$F B$ & 30105 & 20 & $>80001$ \\
\hline HII & $0 .(x)(t)$ & 305 & 2.510 \\
\hline $\mathrm{Fil3}$ & .21 .86 & $>50$ & 2100 \\
\hline Fil & 958.5939 & $>1118$. & $5 \times 101$ \\
\hline
\end{tabular}

TABI.FIV

COMPARISON OF (iPEMLE WITU SAGA-GLS 11$]$

\begin{tabular}{|c|c|c|c|}
\hline Problen & (iPEMIE (B est 1700$)$ & SNCiN.(iL.S IBest $\left.t_{1490}\right)$ & SA(AA-GLLS $H_{1202)}$ \\
\hline$F=1$ & 22.1287 & ot & >कर००1 \\
\hline${ }_{F 10}$ & $\begin{array}{l}0.19090 \\
(10,10477\end{array}$ & s.t.s & $\begin{array}{r}6000 \\
>-x<k(x)\end{array}$ \\
\hline
\end{tabular}

- Bes/,000: the mean of the best function values obtained using 1.000 exact function evaluations by (ilPLML;

- $H_{1,000)}$ : the number of exact function evaluations needed by an algorithm in [1], [10]. [1]] to achieve the sime best function value obtained by (iPLML. In total. (SSSOMA, SAGA-GLS and MAES used $8,(0) 0,6,000)$, and $1,(0)(0)$ exact function evaluations, respectively. If their final results are worse than the GPliML result with 1,00() exact function evaluations. we denote this as $>8.0(0)$. $>0,(00)$, and $>1,000$. respectively.

Some of the above values have not been provided in [1], [10]. [11] in numerical form. so we extract them from the figures in these papers. [10] tried four different prescreening methods and obtained four different results; we choose the best one of them in our comparison.

From Table III to Table $V$, it is elear that GPLME outperforms the three state-of-the-art methods on these test instances. More specifically;

- As shown in Table III, with 1.000 exact function evaluattions for I5, F8, F11 and F13, (BPEML can produce much better results than (GS-SOMA. GS-SOMA is not able to produce solutions with the same quality with 8.000 function evaluations on $\mathrm{F} 5$ and $\mathrm{F} 8$. For $\mathrm{F} / \mathrm{I}$ and $\mathrm{F} 13$, GPEME achieves about two times speed enhancement eompared with GS-SOMA. For I:14, the result obtained by GPLML is also better than (BS-SOMA when both algorithms use 1,000) function evolutions, and GS-SOMA needs about 5.200 function evaluations to obtain similar results.

- The average best function values obtained by GPEML with 1.000 exact function evaluations over 20 runs are better than or similar to those obtained by SAGA-GLS with 6,000 function evaluations as shown in Table IV. The best function values of SAGA-(jLS with 1,000) function evaluations are much worse than those of GPLML.

TABLE V

COMPARISON OF GIPEME WITIT MAES 1$] 0 \mid$

\begin{tabular}{|c|c|c|c|}
\hline Problem & GPENE: (B ESt 1000 ) & MAES I B est 1000 ) & MAES $1 H_{10001}$ \\
\hline $\mathrm{FI}$ & $1.3 e^{-5}$ & 0.1775 & $>1(H(X)$ \\
\hline in & $0.19 \% 0$ & 3 & $>10(x)$ \\
\hline
\end{tabular}

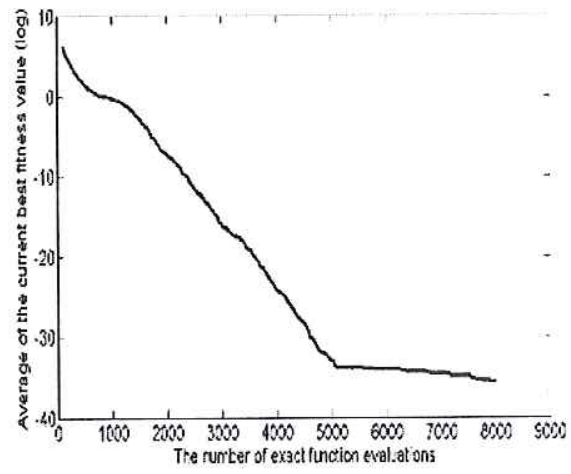

Fig. 3. Convergente curve of the objective function for Fll with 8.000 exact functions evaluations by (il)EMH

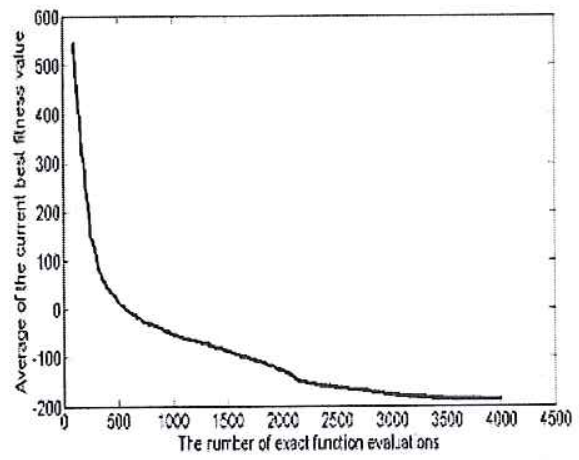

Figs. 4. Convergente curve of the objective lindtion for F13 with f.1000 exact function coaluations by GPEMH:

- MALS also uses 1,0()( function evaluation on Fil and 177. Table $V$ shows that the results obtained by GPLML: are better by one or two orders of magnitude than the results of MALS.

Note that for FIJ and F13, (iS-SOMA can achieve the result of (iPLME in 2.(5)(0)-3.0(0) exact function evaluations. The convergence curves in $|1|$ show that the fitness value decreases very rapidly in (iS-SOMA from 2,000 to 3,000 exact function evaluations. To get an insight into the behavior of GPLML with more than 1,000 function evaluations. we conduct experiments on GPEML: for F11 with 8.000 exact function evaluations and for 11.3 with 4.000 exact function evaluations. The convergence curves of the average best function values found so far over 20 runs are given in lig. 3 and lïg. 4. The average best function values of (iS-SOMA with $8.0(0)$ exact function evaluations are $2.2, \quad 3$ and 126 for [1] and 1:13, respectively $|1|$, while the (iPLML result for [i] with $8.0(0)$ exact function evaluations is $3.333 \quad 16$ and for F13 with 4.000 exact evaluations is 187.04 . Irom Fig. 3 and Fig. 4, it can be seen that the final result of GS-SOMA can be achieved by (iPLML with about 2,000 exact function evaluations. 
TABLE VI

THE EXPERIMENTAL. RESULTS BY THE STANDARD DE FOR F1-F14

\begin{tabular}{c|c|c|c}
\hline Problem & $1,000 \mathrm{FEV}$ & $\mathrm{T}$ & $30,000 \mathrm{FEV}$ \\
\hline $\mathrm{F} 1$ & 203.22 & 12400 & $9.47 \mathrm{e}-17$ \\
$\mathrm{~F} 2$ & 788.05 & 10750 & $4.28 \mathrm{e}-10$ \\
$\mathrm{~F} 3$ & 3281.3 & 5750 & $4.65 \mathrm{e}-4$ \\
$\mathrm{~F} 4$ & 534.85 & 4550 & 1.24 \\
$\mathrm{~F} 5$ & 1621.1 & 5650 & 14.53 \\
$\mathrm{~F} 6$ & 4147.9 & 5800 & 43.70 \\
$\mathrm{~F} 7$ & 16.94 & 9000 & $4.18 \mathrm{e}-9$ \\
$\mathrm{~F} 8$ & 18.35 & 8050 & 0.13 \\
$\mathrm{~F} 9$ & 19.24 & 4100 & 1.18 \\
$\mathrm{~F} 10$ & 81.06 & 11400 & 0.013 \\
$\mathrm{~F} 11$ & 183.18 & 9500 & 0.0025 \\
$\mathrm{~F} 12$ & 467.77 & 5400 & 0.0069 \\
$\mathrm{~F} 13$ & 184.09 & 3150 & -138.15 \\
$\mathrm{~F} 14$ & 995.58 & 1600 & 918.84 \\
\hline
\end{tabular}

The second and last eolumus are the average best function values obtained by DE with 1,000 and 30,000 linction evaluations uver 20 rums. respectively. T is the number of function evaluations required hy the standard DE o achieve similar results by GPEME with 1, 000 linction evaluations.

TABLE VII

COMPARISON BETWEEN (iP +DR AND DIRECT GP FROM FIG. 5 TO FIG. 18

\begin{tabular}{|c|c|c|}
\hline Non, of variathles & (iP+1)R wins & Gl' wins \\
\hline 20 & $1 / 4$ & $3 / 4$ \\
\hline 30 & $1 / 6$ & $5 / 6$ \\
\hline 50 & $4 / 4$ & $(0 / 4$ \\
\hline
\end{tabular}

The second columm shows the number of problems which ean provide a belter result using $\left(\mathrm{GP}^{2}+\mathrm{D}\right) \mathrm{R}$ over the total number of test problems. The third colum shows the same statisties but for the eitse that belter results tim be ohtained by dircet Gil?

\section{Comparison with Stundard DE:}

To understand the benefits of the key techniques in GPLML: we compare (BPEME with the stanclard DE [20] without surrogate model on F1-F14. When applying standard DE, the population size, the sealing factor $F$ and the crossover rate $C R$ are the same as those used in (IPEML: DE/best/ I mutation is also used. The number of exact function evaluations is set to be $30,0(0)$. The experimental results are presented in Table vi.

It is evident from Table VI that GPLME performs much better than DE if the computational budget is 1,000 function evaluations. The efficiency of GPEML comes from the surrogate model-aware search mechanism and the corresponding surrogate modeling method.

\section{E. $($ GP $+D R$ is Direct (iP}

In the above experiments, direct GP modeling is used for the test problems with 20 and 30 variables, and $(\mathrm{BP}+\mathrm{D}$ R for test problems with 50 variables. In the following. we compare the performances of GP+DR and direct (jP modeling in GPEML. Both modeling approaches are tested in GPEML: for I:1 to I:14. Fig. 5 to Fig. 18 compare how the average best function value found so far deceases against the number of exact function evaluations in GPLME with direct GP and GP+DR. The results are based on 20 runs and are summarized in Table VII.

One can observe that:

- For the four problems with 20 variables, (iPLiML with

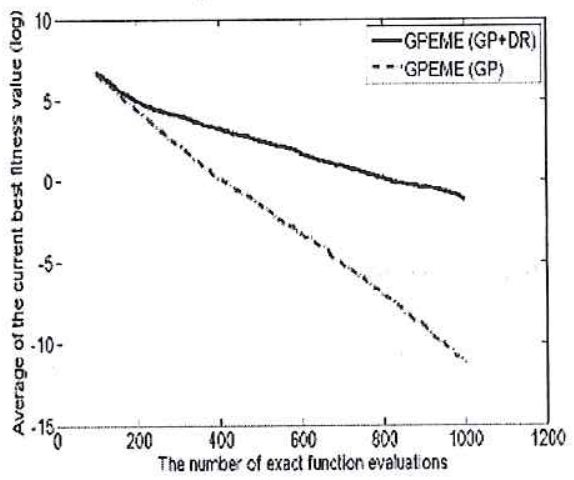

Fig. 5. Convergence curves of the objective function for F1 (20-1) Fllipsoid)

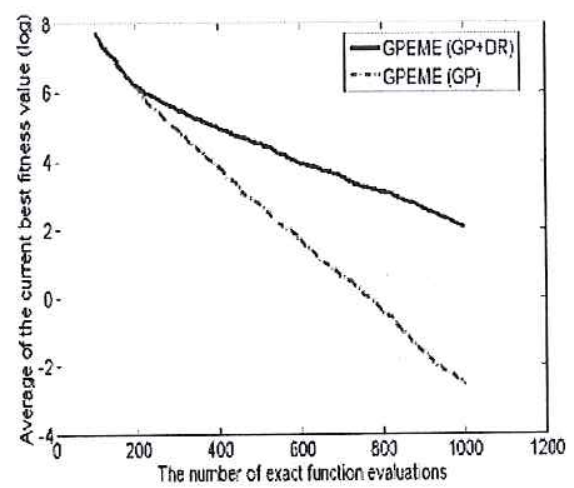

Fig. 6. Convergence curves of the objective function for P2 (30)-1) Ellipsoid

direct (iP elearly performs better than (iP +DR on F1, 177 and $\mathrm{P}^{2} \mathrm{C}$, and $(\mathrm{GP}+\mathrm{D}$ R is a little bit better on $\mathrm{F} 4 \mathrm{4}$.

- For the six problems with 30 variables, (GPLML: with

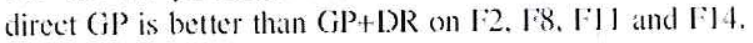
On 155 , (iPLML: with direet (iP performs a little bit better. $(\mathrm{iP}+\mathrm{D}) \mathrm{R}$ wins on FI3.

- On all the four problems with 50 variables, i.e., 13. 1\%. I9) and F12, (BP+DR outperforms direct (il).

Therefore, we can conclude that it is reasonable to use the

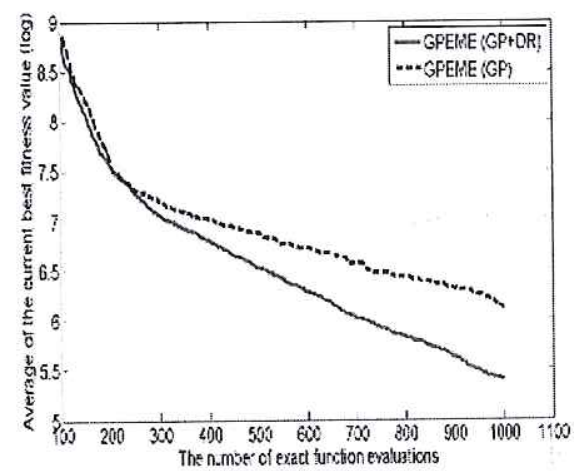

Fig. 7. Comvergence curves of the ohietive function for $1.3(50-1)$ Ellipsoid) 


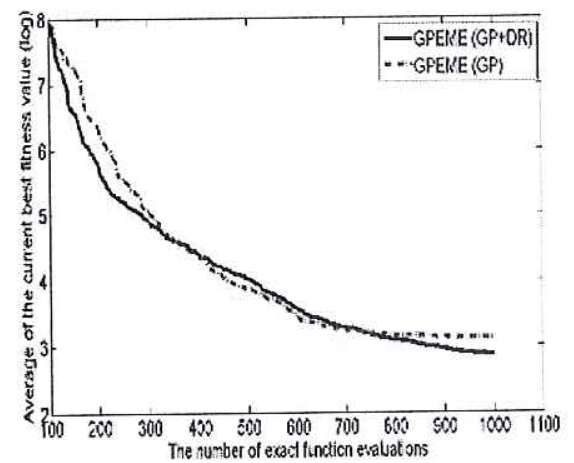

Fịn. 8. Convergence curves of the objective function for F4 (20-D Resenbrock)

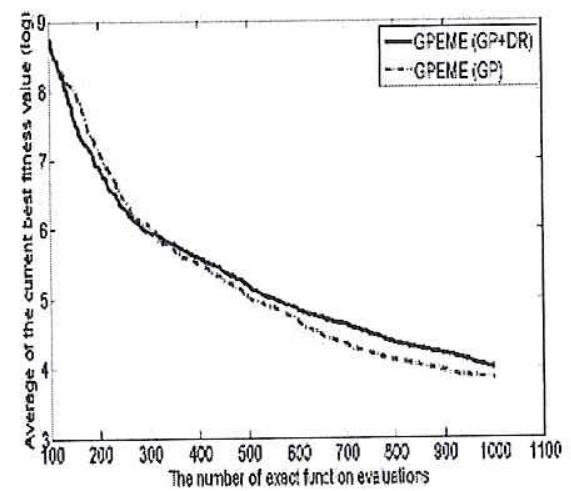

Fig. 9. Convergente curves of the objective function for F5 (30-D) Resenbrock)

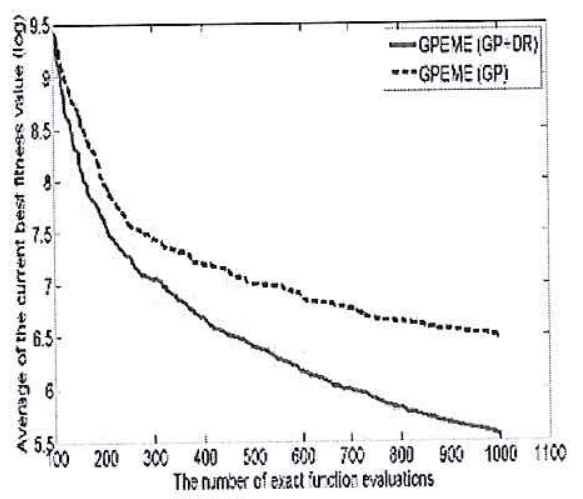

Fig. 10. Convergence curves of the abjective lunction for to (50)-1) Rosenbrock)

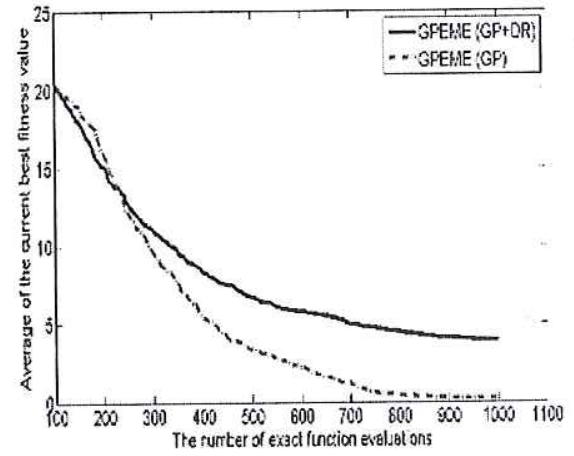

Fig. 11. Compergence curves of the objective function for $\mathrm{F} /(20-1)$ Nekley

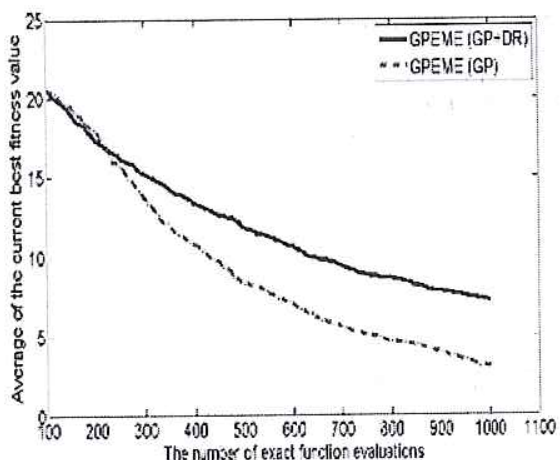

Fig. 12. Comvergente curves of the objective function for 58 (30-1) Ackley)

direct (iP modeling for problems with 30 ) or less variables, and use the $(\mathrm{BP}+\mathrm{D}) \mathrm{R}$ approach for problems with 50 variables.

It is interesting to investigate if the models built by these two approaches are different in terms of quality and if the model quality differences are consistent with the performance differences of GPLME. Recalling that only one model is built and one solution is selected from the $\lambda$ candidate solutions for exact function evaluation in each iteration, it is desirable that the selected solution is one of the best (top-ranked) candidate solutions in terms of exact function values. We divide the $9(0)$ iterations after "Initialization" (i.e., Step 1) into 4.5 phases, each of which contains 20 consecutive iterations. To measure

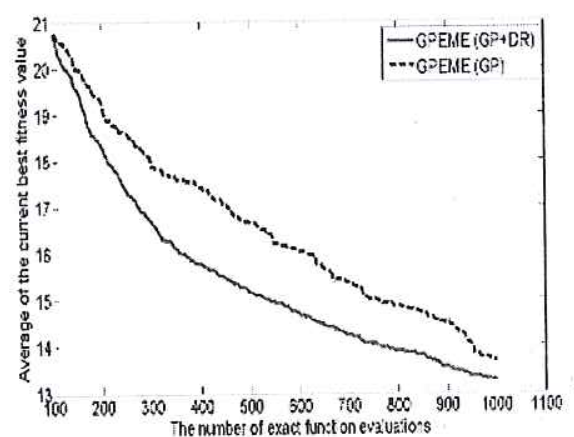

Fig. 13. Comsergence curves of the objective linetion fior Fo) (50-1) Ackley)

(c) 2013 Crown Copyright. Personal use is permitted. For any other purposes, permi ssion must be obtained from the IEEE by em ailing pubs-permissions aieee.org. 


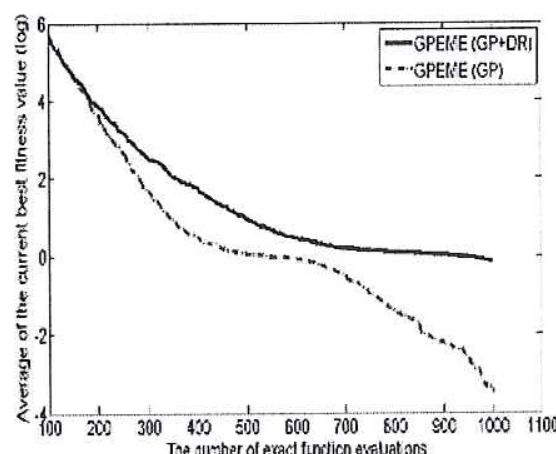

Fig. 14. Convergence curves of the objective lunetion fior F10 (20-1) (jriewink)

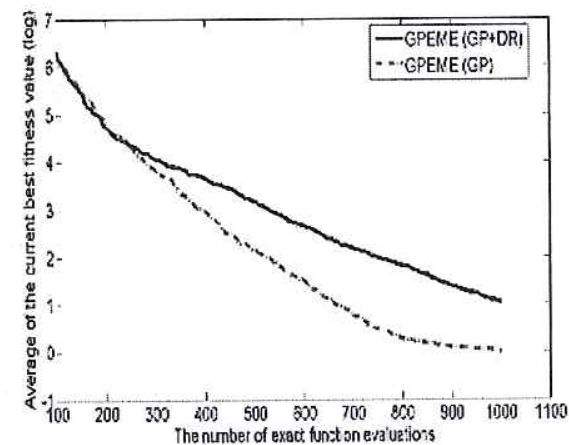

Fig. 15. Convergence curves of the objective lunction lor Fll (30-D) (iriewank)

the quality of modeling in a particular phase. we detine its performance seore as follows:

$$
\text { क } 3 \times N_{1}+2 \times N_{2}+N_{3}
$$

where

- $N_{1}$ is the number of iterations in a phase and in which the selected solution is among the top 2 of the $\lambda$ candidate solutions.

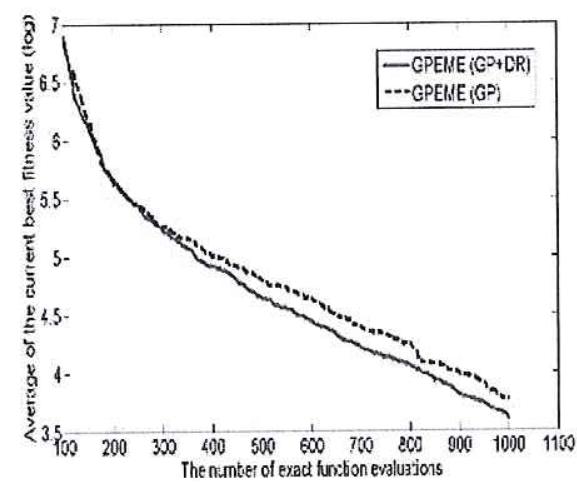

Fig. 16. Convergence curves of the ubjective function for F12 (50-D) Griewank

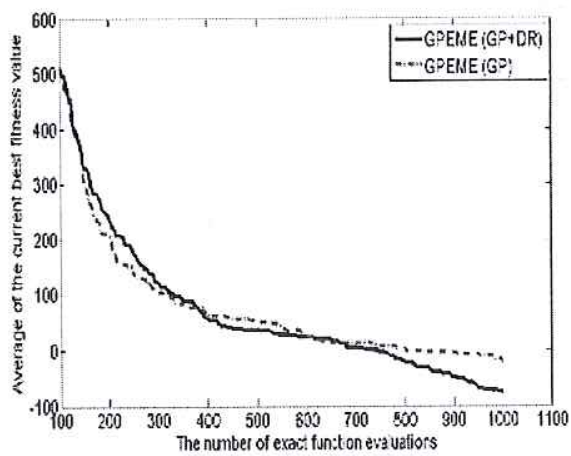

Fig. 17. Convergence curves of the objective lunetion for F13 (301-D) Shifted Rotated Rastrigin

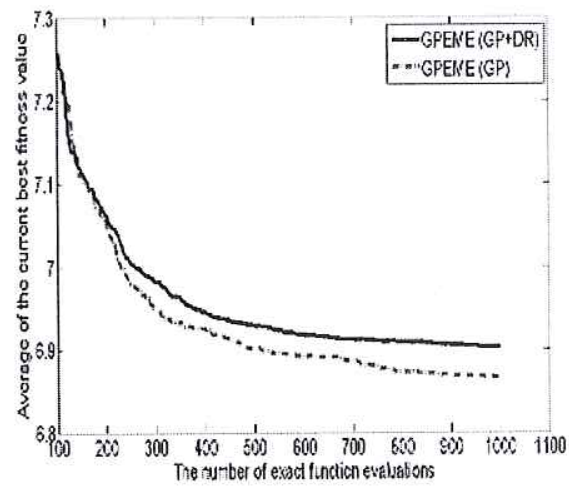

Fig. 18. Convergence curves of the objective function for F14 (30-D) Rataled Hybrid Compesition Fune(ion, F19) in [1]

- $N_{2}$ is the number of iterations in a phase and in which the selected solution is among top 3 to 5 .

- $N_{3}$ is the number of iterations in a phase and in which the selected solution is among top 6 to 10 .

If the selected solution is out of the top 1(). it is not very useful for the search and thus it is not counted in the seore. Clearly. the higher the performance score is. the better the modeling and presereening performs in this phase. Fig. 19 to Fig. 22 plot the seores of two modelings (direct (iP versus (iP + +DR)

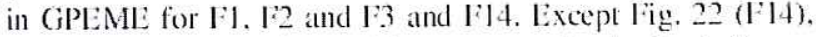
the scores for the other problems are qualitatively similar to

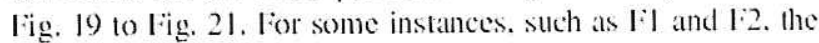
two modeling methods are quite different in terms of scores. More importantly, one can find that the score differences are consistent with the algorithm performance differences. For example, the scores of $G$ P + DR are higher than direct (iP on 13 as shown in lig. 21. and (iPLEE with (BP+DR outperforms the one with direct (jP as shown in rig. 7. The seores of direct (iP are higher on $\mathrm{I} 1$ as shown in Fig. 19. and correspondingly. GPEML with it wins as shown in lïg. 5. P14 is the only exception. But as said above. F14 is an artificially construeted hard problem and it is difficult to optimize. Using the scores. we may be able to improve (iPLML by developing more effective and efficient modeling technigues.

In GPEME. we use Sammon mapping to do dimension 


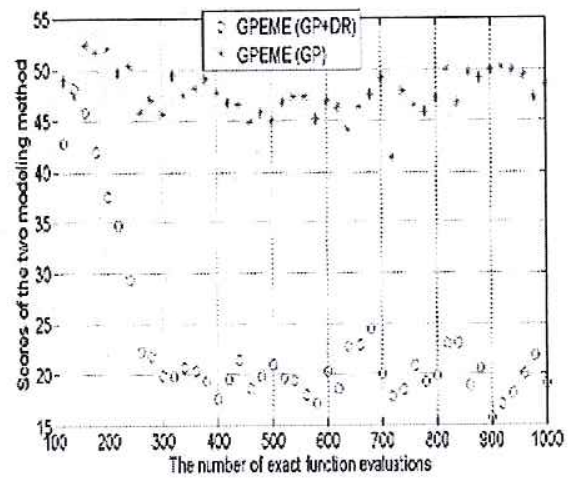

Fig̣. 19. Scores of the (wo modeling approithes for F1 (20-D Ellipsoid)

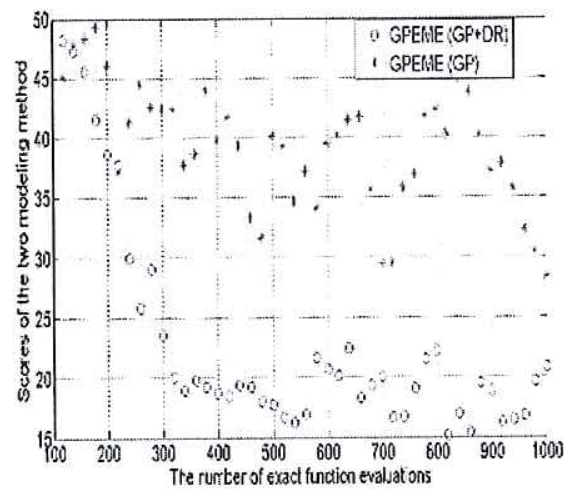

Fig. 20. Scores of the (wo modeling approathes for F2 (30-D Ellipsoid)

reduction. To study the effectiveness of Sammon mapping. some other dimension reduction methods are compared with Sammon mapping in (BPLME on F7. These methods are P'CA. Linear Discriminant Analysis (LDA), Local Linear Limbedding (LLE) and Neighborhood Components Analysis (NCA) [23]. The average best function values found with $1,0(0)$ exact function evaluations over 10 runs are shown in Table Vlll. It ean be seen that Sammon mapping is the best.

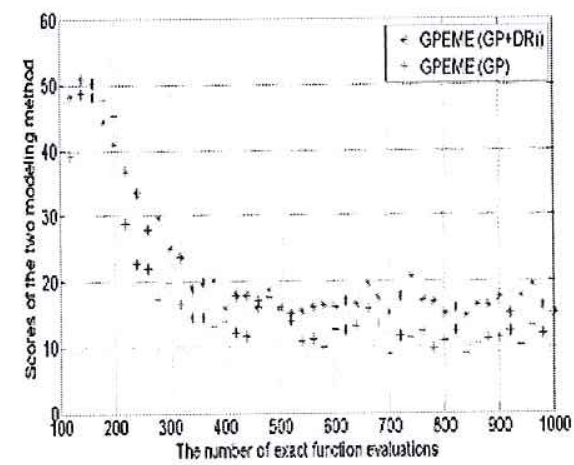

Fig. 21. Seores of the two modeling approathes for F3 (50-D) Ellipsoid)

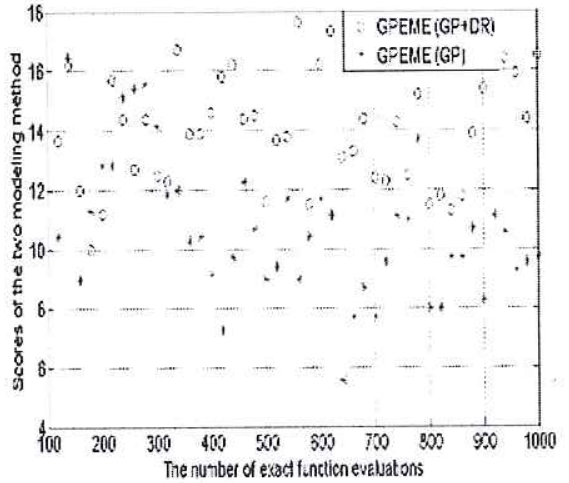

Fig. 22. Seores of the wo nodeling apmoaches for F1t (30-D) Rotated Hybrid Composition Function. F19 in (1)

TABLE VIII

RESULT OF F T USING FIVE DIMENSION REDUCTION METHODS

\begin{tabular}{c|c|c|c|c}
\hline Sammon & PCA & 1.DA & L.LE & NCA \\
\hline 3.57 & 14.93 & 12.5 & 18.73 & 13.29 \\
\hline
\end{tabular}

I: mm-ware Integrated Circuit Optimization

GPLML has been designed for expensive engineering optimization problems. This subsection provides the experimental results of GPEML: for dealing with an mm-wave integrated circuit (IC) design optimization problem. In recent years, highfrequency integrated circuit design is attrating more and more attention, both in academia and industry. At high frequencies. simple equivalent circuit models for passive eomponents are no longer accurate to be used. and a "trial and error" method is inevitably tedious. Therefore. atutomatic design optimi\%ation of mm-wave ICs is of great practical importance. However. electromannetic simulation is required to evaluate candidate designs, which is computationally expensive. Many mm-wave ICs have from 10 to 30 design parameters (i.e., decision variables) to be optimized.

We consider a design optimization problem of a 60 (iH\% 65 nm power amplifier with 17 design parameters. The evaluation of one candidate design requires 1()-12 minutes using AD.S-Momentum, which is a popular electromagnetic simulator. The synthesis time available to this problem is typically one to three days. In this problem. the design parameters are the inner diameters and metal width of the primary and secondary inductors of every transformer. There are three transformers with two inductors in each. The feasible ranges are 20$) \mu m$ to $10(0) m$ for the inner diameter, and $3 / m m$ to 10$) \mu m$ for the metal width. There are also 5 biasing voltages with ranges from $0.5 \mathrm{~V}$ to $2 \mathrm{~V}$. The optimization problem is shown in (12). This is a simulation-based optimization problem. so no explicit analytical formulations for its objective and constraints are available.

(c) 2013 Crown Copyright. Personal use is permitted. For any other purposes, permission must be obtained from the IEEE by emailing pubs-permissions@ieee.org. 


\section{REFERENCES}

11] D. Lim, Y. Jin, Y. Ong. and B. Sendholit, "Generalizing surngateassisted evolutionary computation." IEEE Transacrions on Evolutionary Computution, vol. 14, 130. 3, pp. 329-355. 2010.

|2| J. Kuwwles. "IarE(GO: $A$ hybrid algorithm with on-line landscape approximation for expensive multiobjective eptimization problems," IEEE Transections on Erolationary Computation, vol. 10, no. 1. pp. 50-66, 2006.

13] D. Allstot, K. Choi, and J. P'ark, Parasitic-aritare oprimi:ation of CMOS $R F$ circuits. Springer Netherlands. 2003.

14) T. Milligan and J. Wiley, Motern antenna design. Wiley Online Library. 2005.

IS| L. Mercado, S. Kuo, T. Lete. and R. L.ee, "Analysis of RF MEMS switch patckaging process for yicld improventent," IEEE Transactions on Adranced Packaging. vol. 28. 110. 1, pp. 134-141, 2005.

[6] $\mathrm{H}$. Chan and P. Fnglert, Accolereted sams resing handhonk. IEEE Press, 2001.

17| D. Jones. M. Schonlatu, and W. Welch. "Eflicient global optimization of" expensive black-box lunetions," Asternal of Cilohal opminization, vol. 13. 116. 4. pp. $45.5-492.1998$.

18) Y. Jin, "A Lomprehensive survey of' lituess approximattion in evolutionary computation," Soft Compuning-A Fusion of Fondandons, Methodologias and Applications, vol. 9. no. 1. pp. 3-12, 2005.

(9) B. Litu, D. Zhio, P. Reynatert, and (i. Cielen, "Synthesis of integrated passive components lor ligh-frequeney RF ICS bised on evolutionary computation and machine learning techniques," IETE Transations on Computer-Aided Design of Integrated Circuits and Sixtems, vol. 30, no. 10, pp. $1458-1468.2011$.

[10] M. Fnmerich. K. Giamakoglou. and B. Naujoks, "Single-ind muliobjective evolutionsary optimization assisted by Gaussian ramstom field metamedels." IEEE Transations on Evolutionary Compunation, vol. 10. 110. 4. 1). $421-439,2006$

111] Z. Zhou. Y. Ong, P. Nair, A. Keane, and K. L.mo. "Combining global and local surrogate models (o accelerate evolutionary optimization," IEFE Tremsetions on Systems. Men, and Cybernetics. Pat C: Applications and Roviens, vol. 37, no. 1, pp. 66-76. $200 \%$.

$112 \mid$ D. Jones, "A taxomomy of" global optimization methods based on response surfaces," Jommal of Glohal Optimization, vol. 21, no. 4. pp. $34.5-38.3,2001$.

113] K. Giammakoglou, "Design of optinal aerodymamic shapes using stochastic optimization methods and computational intelligence." Progress $i$ Aernspace Seiences, vol. 38, no. 1, pp. 43-76, 2002.

(14) Y. Ong, K. Y. Lum, and P. Nair. "Hybrid evolutionary algorithm with hermite radial basis funtion interpolants lor computationally expensive adjoint solvers," Compuntational Optimianion and Applications, vol. 39. no. $1, \mathrm{pp} .97-119,2008$.

135| Q. Thang, W. Liu. E. Tsang, and B. Virginas, "Expensive nultiohiective optimization by MOEND with Gaussian process model." IEEE Tirumsactions on Erolutionury Computution, vol. 14, no, 3, pp. 456-474. 2010.

[16] I. Vuutchkov and A. Keanc, "Multi-objective optimization using sumogates." Computational Imelligence in Optimization. pr. 155-175, 2010

$117 \mid$ J. Demis and V. Torean. "Managing approsimation models in optimization," Multidisciplinary design optimization: State-st-the-art, pp. 330-347, 1997.

[18] E. Ackermmus, J. de Villiers, and P. Cilliers, "Nonlinear dynamic systems nodeling using Gatussian processes: Predicting ionospheric totil electron eontent over south afriea." J. Geophyx. Res, vol. 116, no. Al0. p. A10303, 2011.

[19] D. Buche, N. Schraudolph, and P. Kounoutsakus, "Accelerating cwolutionary algorithms with Gatussian process lituess function models." IEF: Transetrions on Systems. Mfan, and Cybernetics, Part C: Applicutions and Reviews, vol. 35, no. 2, pp. 183-194. 2005.

120| D. Gorissen, I. Couckuyt, P. Demeester, T. Dhacne, and K. Crombece i) surrogate modeling and adaptive sampling toolhox for computer

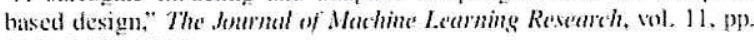
$2051-2055,2010$.

1211 C. Rasmussen. "Gaussian processes in mathine learning," Adranced Lecturess on . Aachine Lecuming, pp. 63-71, 2004.

122] J. Sacks. W. Welch. T. Mitchell, and H. Wym. "Design and antysis of computer experintents," Statisfical science, vol. 4. no. 4. pp. 409-423 $198 \%$.

1231 1.. Mataten. E. Postma, and H. Herik. "Dimensionality reduction: A comparative review." Publishad miline, vol. 71, no. Jamuary, pp. 25962603. 2008 .

$24 \mid$ J. Sammon Jr, "A nonlintar mapping lor data structure amalysis," IEEE Transections on Computers. vol. 100, no. 5, pp. 401-409. 1960.
|25| M. Avriel, Nonlinear programming: andysis and molhods. Dower Puhns. 2003.

126] K. P'riee, R. Stom, and J. Lampinen. Differential crolation: a pratical approuch to global optimization. Springer-Verlag New York lnc, 2005.

[2\%] M. Stein, "Large sample properties of" simulations using litun hypercube sampling." Tichemetrics, vol. 29, no, 2, pp. 1433151, $198 \%$

$28 \mid$ P. Suganhan, N. Hansen, J. Liang, K. Deb. Y. Chem, A. Auger, and S. Tiwari. "Problem delinitions and eviluation eriteria for the CEC 2005 special session on real-paraneter optimi/ation." Nemyang Ticdmological Unirervity; Singepon?, Tech. Rep, vol. 20050015. 2005 .

|29| D. Zhato, Y. He. L. L.i. D. Joos, W. Philibert, and P. Reynatert. "A fot (iHz 14 dBm power amplifier with at tras former-based power combine

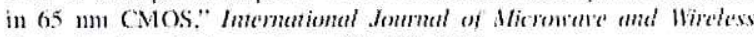
Techologies. vol. 3. nu. 2. pp. 90-105, 2011.

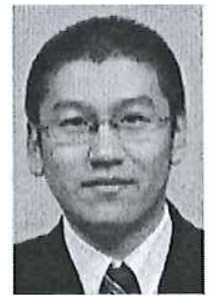

Bo Liu received the B.S. degree from Tsinghua Universily: P' R. Chinis in 2008 . He received his Ph.D. degree at the MICAS laboratories of the Katholicke Universiteit Leuven, Be gigim, in 2012.

$\mathrm{Hc}$ is currently i lecturer (Assistant Prolessur) with Department of Computing. Gilyndwr (Jniversity, U.K. From Octuber 2012 (1) March 2013, $x$ is : Humboldt research fellow and is working with Technical University of Dormund and Techuical (niiversity of Munich, Germbny. His research interest: lic in evolutionary computation. mathine learning. luzsy logic and design attontation methodologics of ansalog / RF integrated circuits and antemas. $\mathrm{He}$ has authored or soathored 1 boxk and more than 20 papers in intemational journals and conlerence procedings.

Dr. Liu was at session organizer on computationally expensive optimization and optimization in uncertain enviromments in IFFE World Congress on Computational Intelligence 2012. He is in the editurial board or ans a reviewe in artificial intelligence and elcetronic design atutomation liclds, such as II:FE Transactions on Evolutionary Computation. IFEE Transictions on ComputerAided Desion ol lntegrated Circuits and Systems, Jnumal of Engineering. He is also a book reviewer of Elsevier and Bentham Sciente P'ublishers.

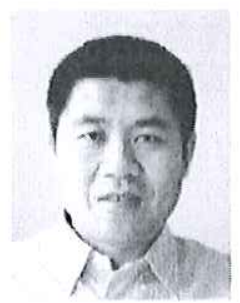

Qingfu Zhang (M'0I-SM'or) received the BS in mathentatics from Shamsi University. China in 1984, the MSc in applied mathentatics and the PhD in infornation enginecring frous Xidian Unversity. China. in 1991 and 1994. respectively.

He is currenty a Professor with the School of Computer Science and Ficctronic Enginecring, University of Fssex. UK. From 1994 to 2000 . he was with the National Laboratory ol' P'arallel l'rocessing and Computing. Natimal University of Defence Sciente and Techuology. China. Hong Kong Poly cechuie University. Hong Kong, the Geman National Research Centre for Infombation Tedhulogy (now Framboler-(iesellschaft. Gernany), and the University of Manchester lnstitute of Science and Techuology. Manchester. U.K. He holds two patents and is the athor of mimy researeh publitations. His min research interests include evolutionary computation. optinization. neural networks, data analysis, and their appliciations.

Dr. Zhang is an Associate Editor of the IEtEF Trimsattions on Evolutionary Computation and the IEFE Tramsatedions on Systems. Man. and Cyberneticsic Part B. He is also an Editorial Board Member of thee other international journals. MOFADI), a multobjevitwe optimization algerithm developed in his group, won the Uncenstrained Multiobjective Optimization Algorithm Competition at the Congress of Evolutionary Computation 2009), and was atwarded the 2010 IEEE Transactions on Evolutionary Computation Outstanding Paper Award. 


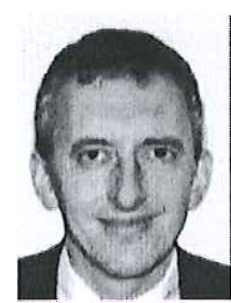

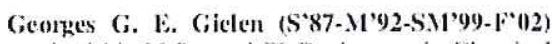
received his M.Sc. and Ph.D. degrees in Electricil Engincering from the Katholicke Universiteit L.euven, Belgium, in 1986 and 1990, respectively. In 1990. he was appointed als a posidoctoral research assistant and visiting lecturer at the department of Electrital Fingineering and Computer Science of the University of Cadifornia, Berkeley. In 1993, he was appointed assistimt prolessor at the Katholieke Universiteit L_cuven, where he was promoted to full professur in 2000.

His research interests are in the design of imalog and mixed-signal integrated circuits, and especially in analog and mixed-signal (AD) (ools ind design automiation (modeling. simulation and symbolic analysis, analog synthesis, analog layout gencration, amalog and mixed-signal testing). He has ituthored or coatuthored wo books and more than 400 papers in edited books. internitional jountals and conference protectings. He received the 1995 Best Paper Award in the John Wiley international journal on Círeut Theory and Applieations, and was the 1997 Latureate of the Belgian Royal Acadeny on Sciences, Litenature and Arts in the discipline of Engineering. He received the 2000 Aleatel Award from the Belgim National Fund of Scientific Research For his inmovative research in telecommunications, and won the DATF. 2004 Best laiper Award. He is a Fellow of the If:EE. served as elected member of the Bourd of Governors of the IEEE. Circuits And Systems (CAS) socicty and

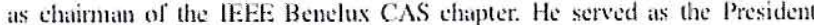
of the IEFFE Circuits And Systems (CASi Society in 2005. 